\title{
Design and Implementation of a Non-invasive and Cuff-less Arterial Blood Pressure Monitoring System
}

\author{
Seyed Mohsen Anvari ${ }^{1}$, Mohammadreza Yazdchi ${ }^{1}$, Amirhossein Kayvanpour ${ }^{1}$, Seyed Mohammad \\ Hasan Nayebpour ${ }^{1}$, Tero Koivisto ${ }^{2}$, Mojtaba Jafari Tadi ${ }^{2 *}$ \\ ${ }^{1}$ University of Isfahan, Department of Biomedical Engineering, Iran \\ ${ }^{2}$ University of Turku, Department of Future Technologies, Finland
}

\begin{abstract}
Hypertension or high blood pressure $(B P)$ is one of the most common worldwide disease leading to heart attack or stroke. Continuous assessment of blood pressure level is key to diagnosing hypertension. In this study, we designed and tested a dedicated cuff-less monitoring system which estimates BP level without need for calibration. We obtained continuous measurements from 40 healthy subjects (30 males and 10 females) ranging from 20-30 years old. Our measurement protocol consisted of 15 minutes simultaneous electrocardiography (ECG) and photoplethysmography (PPG) within three sessions, i.e. rest, bicycle exercise, and recovery. From ECG and PPG signals, we obtained 34 candidate features from which up to 9 features were selected to estimate systolic and diastolic BP levels. We validate our results with three regression models such as linear regression, support vector machines (SVM) regression, and multilayer perceptron (MLP) to obtain the best results. The study provides a promising approach for modern cuff-less BP monitoring devices.
\end{abstract}

\section{Introduction}

According to the American Heart Association 2017 statistics, heart diseases are the number one cause of death globally. Cardiovascular diseases (CVD), which are a group of disorders of the heart and vessels, are considered as the leading cause of death for $30.8 \%$ of all deaths in the United States in 2014 [1]. Hypertention or high blood pressure (HBP) is a major cardiovascular risk factor for CVD and stroke that is curable, but requires accurate and reliable detection and control strategies [1]. Continuous assessment of blood pressure (BP) level is one way to improve hypertension detection by providing serial measurements in the mass population [2]. Therefore, it is imperative to have an effective strategy to accurately and safely diagnose at-risk subjects for early intervention and fast response to sudden and potentially lethal events.
Several invasive and non-invasive BP measurement methods are commonly used in clinical sites. Catheterization is the golden standard to monitor instantaneous arterial blood pressure, which requires invasive interventions to measure accurate BP from central venous sites [3]. Auscultation, oscillometry, and volume clamping are the other most popular noninvasive cuff-based methods that measure systolic and diastolic BP based on different configurations. Auscultation is based on sphygmomanometer and detecting Korotkoff sounds using a stethoscope, while oscillometry is an automatic method based on pressure sensors inside a cuff which may be fitted on the wrist [4]. Volume clamping is a recently developed approach to automatically measure instantaneous BP using a photoplethysmography (PPG) sensor attached to the finger and a inflating-deflating cuff fasten around arm [5]. However, cuff-based BP measurement methods are cumbersome, time consuming, inconvenient, and troublesome during ambulatory monitoring, particularly for long term continuous monitoring.

\section{Related Work}

Several modern approaches for cuffless BP monitoring and noninvasive and automated cardiovascular monitoring are now available. New methods are mostly based on waveform measurement from ECG, PPG sensor, bioimpedance (EBI)/impedance cardiography (ICG), ballistocardiography, and videoplethysmography (VPG) as they aim to measure pulse wave velocity (PWV) [6], pulse arrival time (PAT), and pulse transit time (PTT) [7] indexes as surrogate indicators of blood pressure. However, such methods pose a great challenge which is calibration of PTT/PWV (in units of $\mathrm{m} / \mathrm{s}$ or $\mathrm{ms}$ ) to BP (in units of $\mathrm{mmHg}$ ) [4].

In this study, we designed a non-invasive cuff-less prototype for continuous and long term blood pressure monitoring which requires no calibration. Our proposed method is based on feature extraction from simultaneous electrocardiography (ECG) and PPG signals. We considered the use 
of machine learning techniques, mainly regression models, to estimate systolic and diastolic BP with different measurement protocols. This paper is organized as follows: Section 3 describes details of our designed data acquisition system, experimental set-up, and protocols employed for data collection and analysis. Section 4 describes biosignal preprocessing, followed by feature extraction and selection pipelines for machine learning experiments. Section 5 delineates experimental results, Section 6 discusses the limitation and future direction of this work, and finally concludes this work.

\section{Materials and Methods}

The data acquisition for this study consists of 40 recordings, each of a duration of a 45 minutes, taken from healthy volunteers (30 males and 10 females ranging from 20-30 years old) with their written informed consent. The research protocol was approved by the the institutional review board of University of Isfahan and performed according to the Declaration of Helsinki.

Our measurement protocol consisted of continuous 15 minutes blood pressure monitoring within three sessions, i.e. rest, bicycle exercise, and recovery, with a 3 minutes intersession pause. In the first session, subjects were asked to lie on supine position for 15 minutes. Subsequently, they were asked to perform 15 minutes work out using an exercise bike, and finally 15 minutes recovery while seating on a chair. A dedicated wearable Holter monitoring system was designed to capture concurrent ECG (three lead Holter device, AD8236, Analog Devices, USA) and PPG finger sensor (Nellcor pulse oximetry, USA) with a sampling rate of $1024 \mathrm{~Hz}$ and a resolution of 16 bits. Additionally, a custom-made software was developed for realtime acquisition of signals to be stored on .wav format in a host computer. A reference cuff-based automatic blood pressure monitor (Omron MX3 Plus, Japan) was used for validation purposes. All signal processing and feature extraction tasks were made using Matlab software while machine learning experiments were accomplished in WEKA environment.

Level of blood pressure was measured as two numbers: systolic blood pressure (SBP) - pressure caused by blood against artery walls when the heart beats - and diastolic blood pressure (DPB) - the pressure in the vascular system when the heart is relaxing. Blood pressure evaluations were made according to Association for the Advancement of Medical Instrumentation (AAMI) and British Hypertension Society (BHS) protocols.

\section{Machine Learning Pipeline}

\subsection{Feature Extraction}

After collecting data, we extracted 34 candidate features from the measured ECG and PPG signals. Primary pre-processing pipelines started with motion artifact removal followed by bandpass filtering of ECG (0.02-43 $\mathrm{Hz}$ ) and PPG (0.01-20 Hz) for noise and baseline wandering removal. ECG R-wave peaks were detected using an adaptive low-complexity QRS detector method [8]. Afterwards, PPG peak and valley locations were identified to measure time intervals. The local maxima (systolic point) in PPG signal is considered as the peak between the interbeat R-waves, while the local minima (diastolic point) is considered as the first down-ward wave before the local maxima.

Our extracted features are based on the time intervals between the ECG $\mathrm{R}$ and location of maxima and minima points in PPG signal. These features can be divided into two groups. The first group of features refers to the timings between the R-wave and PPG points to measure pulse parameters (feature 1-6), while the latter group considers PPG pulsatile components and its derivations in time domain (7-34). Figure 1 shows an overview of the feature extraction from PPG pulsatile components and Table 1 represents a full description of all extracted features in this study.

Time intervals between respectively 10, 25, 32, 50, 66, and 75 percent of the PPG amplitude - upward slope from diastolic to systolic points - are nominated by - diastolic width (DW) - DW10, DW25, DW32, DW50, DW66, and DW75, while for the PPG downward slope they are nominated by - systolic width (SW) - SW10, SW25, SW32, SW50, SW66, and SW75 [9]. We did not consider features related to the dicrotic notch because this peak is imperceptible in some cases, specifically elderly subjects, may fully be disappeared during exercise.

\subsection{Feature Selection}

After obtaining 34 candidate features, we performed a two-step feature selection approaches based on calculating correlation coefficient $(r)$ in order to identify best features in terms of regression power for estimating blood pressure. Correlation coefficient value was calculated as:

$$
r=C(X, Y) / \sqrt{D(X) \cdot D(Y)}
$$

Where $X$ is one set of the 34 extracted candidate features and $\mathrm{Y}$ is one of the two pressures, systolic or diastolic. $C$ donates covariance between two variables and $D$ donates variance. In the first method, for each subject we considered an average value for the measured BPs (systolic 

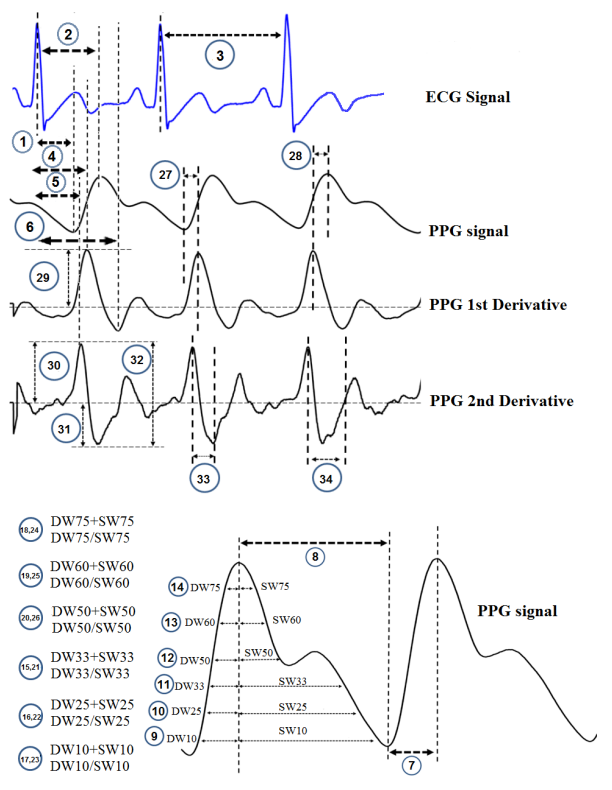

\begin{tabular}{|l|l|}
\hline Number & \multicolumn{1}{c|}{ Feature Description } \\
\hline 1 & Time delay between ECG R and PPG diastolic point \\
\hline 2 & Time delay between ECG R and PPG systolic point \\
\hline 3 & Time interval between ECG R peaks \\
\hline 4 & Time delay between ECG R and PPG 1st derivative local maxima \\
\hline 5 & Time delay between ECG R and PPG 2nd derivative local maxima \\
\hline 6 & Time delay between ECG R and PPG 1st derivative local minima \\
\hline 7 & $\begin{array}{l}\text { Time delay between PPG disatolic and following systolic point } \\
\text { (systolic upstroke time) }\end{array}$ \\
\hline 8 & $\begin{array}{l}\text { Time delay between PPG systolic and following diastolic point } \\
\text { (diastolic time) }\end{array}$ \\
\hline $9-14$ & DW10, DW25, DW33, DW50, DW66, DW75 \\
\hline $15-20$ & $\begin{array}{l}\text { DW33 + SW33, DW25 + SW25, DW10 + SW10, DW75 + SW75, } \\
\text { DW66 + SW66, DW50 + SW50 }\end{array}$ \\
\hline $21-26$ & $\begin{array}{l}\text { DW33 / SW33, DW25 / SW25, DW10 / SW10, DW75 / SW75 } \\
\text { DW66 / SW66, DW50 / SW50 }\end{array}$ \\
\hline 27 & $\begin{array}{l}\text { Time delay between PPG diastolic point and PPG 1st derivative } \\
\text { local maxima }\end{array}$ \\
\hline 28 & $\begin{array}{l}\text { Time delay between PPG systolic point and PPG 1nd derivative } \\
\text { local maxima }\end{array}$ \\
\hline 29 & Amplitude of PPG from baseline to the 1st derivative local maxima \\
\hline 30 & Amplitude of PPG from baseline to the 2nd derivative local maxima \\
\hline 31 & Amplitude of PPG from baseline to the 2nd derivative local minima \\
\hline 32 & Amplitude of PPG 2nd derivative local minima to maxima \\
\hline 33 & Time delay between PPG 2nd derivative local maxima and minima \\
\hline 34 & Time delay between PPG 2nd derivative local maxima and baseline \\
\hline
\end{tabular}

Figure 1. Feature extraction from PPG pulsatile components.

Table 1. List of features from ECG and PPG signals.

and diastolic) as well as candidate features ( 3 values for the pressures and 3 candidate feature set were obtained during the rest, exercise, and recovery sessions). Afterwards, the correlation coefficient, $r$, was calculated between the measured reference systolic-diastolic blood pressures and the mean value of the extracted features. Candidate features with the correlation coefficient greater than $|0.6|$ with both systolic and diastolic BPs were selected in this step.

With the second method, we considered the correlation between the blood pressures and each extracted feature for all subjects, and subsequently selected features of which have the correlation greater than $|0.6|$ with both systolic and diastolic pressure. Finally, selected features which are common in both methods were selected for regression analysis tasks.

\subsection{Learning and Cross-validation}

After selecting suitable features, we performed different methods to estimate systolic and diastolic blood pressures. We considered the use of three regression models such as linear regression, support vector machines (SVM) regression, and multilayer perceptron (MLP) to obtain the best results. In order to evaluate each of these methods, we assessed three metrics, i.e. mean absolute error (MAE), standard deviation (SD), and correlation coefficient between the true measured values and estimated numbers by the regression models. In order to avoid possible over-fitting we considered a 10 -fold cross validation for evaluating our learning approaches

With the MLP method, we tested various number of hid- den layers and neurons and finally obtained best results with 1 hidden layer and 4 neurons for systolic pressure and 2 hidden layers and 3 neurons for the diastolic pressure estimations. The SVM learning algorithm was trained with radial basis function (RBF) kernels.

\section{Results}

Table 2 shows the performance of the three regression models by assessing MAE, SD, and correlation coefficient (CC) of the estimated systolic and diastolic BP levels. The average MAE and SD were $5.61 \mathrm{mmHg}$ and $4.86 \mathrm{mmHg}$ for systolic $\mathrm{BP}$ and $5.32 \mathrm{mmHg}$ and $4.27 \mathrm{mmHg}$ for diastolic BP, respectively. As can be seen from Table 2 among the learning methods, KSVM and MLP represented very close performances. However, due to the fact that MLP is less computationally demanding we considered it for the final implementation of the system. Therefore, we compared results obtained from MLP method against BHS and AAMI standards for greater certainty. According to the BHS standard, the reliability of BP estimations can be divided into three categories: $\mathrm{A}, \mathrm{B}$, and $\mathrm{C}$. For example, in category $\mathrm{A}$, at least $60 \%, 85 \%$, and $95 \%$ of the estimated cases have a difference less than $\pm 5, \pm 10$, and $\pm 15 \mathrm{mmHg}$ of standard value.

Table 3 shows the results obtained from MLP approach as compared to the BHS standards. When compared to the AAMI requirement, the estimated blood pressures should have error and standard deviation rates less than $5 \mathrm{mmHg}$ and $8 \mathrm{mmHg}$, respectively. Thus, for the MLP method, the average error rate for the systolic and diastolic BP were 
-1.5 and $-0.2 \mathrm{mmHg}$, while the standard deviations were 6.93 and $6.65 \mathrm{mmHg}$, respectively.

Table 2. Performance assessment of regression models for BP estimation

\begin{tabular}{|c|c|c|c|c|c|c|}
\hline \multirow{2}{*}{ Model } & \multicolumn{3}{|c|}{ Systolic BP } & \multicolumn{3}{c|}{ Diastolic BP } \\
\cline { 2 - 7 } & $\begin{array}{c}\text { MAE } \\
(\mathrm{mmHg})\end{array}$ & $\begin{array}{c}\text { SD } \\
(\mathrm{mmHg})\end{array}$ & $\begin{array}{c}\mathrm{CC} \\
\left(\mathrm{r}^{2}\right)\end{array}$ & $\begin{array}{c}\text { MAE } \\
(\mathrm{mmHg})\end{array}$ & $\begin{array}{c}\text { SD } \\
(\mathrm{mmHg})\end{array}$ & $\begin{array}{c}\mathrm{CC} \\
\left(\mathrm{r}^{2}\right)\end{array}$ \\
\hline Linear regression & 5.83 & 4.83 & 0.86 & 5.68 & 4.30 & 0.63 \\
\hline SVM regression & 5.55 & 4.42 & 0.88 & 5.33 & 4.19 & 0.67 \\
\hline MLP & 5.46 & 4.79 & 0.87 & 4.96 & 4.32 & 0.70 \\
\hline
\end{tabular}

Table 3. MLP performance with respect to the BHS standards

\begin{tabular}{|c|l|l|l|l|}
\hline \multicolumn{2}{|c|}{} & $5 \mathrm{mmHg}<$ & $10 \mathrm{mmHg}<$ & $15 \mathrm{mmHg}<$ \\
\hline \multirow{2}{*}{ MLP } & Systolic & $55 \%$ & $86 \%$ & $97 \%$ \\
\cline { 2 - 5 } & Diastolic & $53 \%$ & $89 \%$ & $97 \%$ \\
\hline \multirow{2}{*}{$\begin{array}{c}\text { BHS } \\
\text { Standard }\end{array}$} & Category A & $60 \%$ & $85 \%$ & $95 \%$ \\
\cline { 2 - 5 } & Category B & $50 \%$ & $75 \%$ & $90 \%$ \\
\cline { 2 - 5 } & Category C & $40 \%$ & $65 \%$ & $85 \%$ \\
\hline
\end{tabular}

\section{Discussion and Conclusion}

In this study we presented a cuff-less and non-invasive blood pressure monitoring system which is solely based on features extracted from simultaneous ECG and PPG signal processing and neural network. Our designed system offers accurate and reliable estimation of systolic and diastolic blood pressure values without need for calibration in advance. The final system is based on a real-time data gathering software powered by advanced signal processing and machine learning algorithms for accurate measurement of the blood pressure. Additionally, in order to have a comprehensive and operational device for all type of subjects including heart diseased patients, we designed a small-size wearable Holter system which is connected with an optical sensor and a running software.

The main limitation of this research is that heart diseased patients were excluded and only 40 young healthy subjects were examined; this effects the robustness and statistical power of our outcomes. However, the results are encouraging, and warrant subsequent measurements and analysis with a bigger study group including heart diseases subjects. Our future direction would include evaluation of our method against invasive blood pressure monitoring systems with both healthy and diseased subjects. Furthermore, a new miniaturized hardware system will be developed for wearable physiological data recording. Noninvasive physiological monitoring is an area of high interest with potential for significant patient benefit. Allowing early detection and prediction of cardiovascular diseases, such as hypertension, will affect patient outcome and quality of life after treatment. Additionally, user-friendly and cost-effective monitoring systems will enhance the quality of treatment and daily life quality of cardiac patients.
In conclusion, the achieved results in this study are consistent with BHS and AAMI standards which confirm the accuracy of the measurements obtained by the designed BP monitoring system.

\section{Acknowledgements}

This work was supported in part by the Iranian Ministry of Science and Research graduate grant and Academy of Finland under Grant 290930.

\section{References}

[1] Benjamin EJ, et al. Heart disease and stroke statistics-2017 update: A report from the american heart association. Circulation 2017;ISSN 0009-7322.

[2] Pickering TG, Shimbo D, Haas D. Ambulatory bloodpressure monitoring. New England Journal of Medicine 2006;354(22):2368-2374. PMID: 16738273.

[3] McGhee BH, Bridges EJ. Monitoring arterial blood pressure: What you may not know. Critical Care Nurse 2002;22(2):60 79.

[4] Harrington GR, Hnatilik OW. Noninvasive monitoring. The American Journal of Medicine 1993;95(2):221 - 228. ISSN 0002-9343.

[5] Mukkamala R, Hahn JO, Inan OT, Mestha LK, Kim CS, Treyin H, Kyal S. Toward ubiquitous blood pressure monitoring via pulse transit time: Theory and practice. IEEE Transactions on Biomedical Engineering Aug 2015; 62(8):1879-1901. ISSN 0018-9294.

[6] Gribbin B, Steptoe A, Sleight P. Pulse wave velocity as a measure of blood pressure change. Psychophysiology 1976; 13(1):86-90. ISSN 1469-8986.

[7] Geddes LA, Voelz MH, Babbs CF, Bourland JD, Tacker WA. Pulse transit time as an indicator of arterial blood pressure. Psychophysiology 1981;18(1):71-74. ISSN 1469-8986.

[8] Gutirrez-Rivas R, Garca JJ, Marnane WP, Hernndez . Novel real-time low-complexity qrs complex detector based on adaptive thresholding. IEEE Sensors Journal Oct 2015; 15(10):6036-6043. ISSN 1530-437X.

[9] Kurylyak Y, Lamonaca F, Grimaldi D. A neural networkbased method for continuous blood pressure estimation from a ppg signal. In 2013 IEEE International Instrumentation and Measurement Technology Conference (I2MTC). ISSN 1091-5281, May 2013; 280-283.

Address for correspondence:

Mojtaba Jafari Tadi,

University of Turku, Finland

mojtaba.jafaritadi@utu.fi 